\title{
Spectrum of Scedosporium apiospermum infections in a tertiary care centre in North India: Experience over three years
}

Authors: Gagandeep Singh ${ }^{1}$, Immaculata Xess ${ }^{1}$, Reshu Agarwal ${ }^{1}$, Suneeta Meena ${ }^{1}$, Janya Sachdev ${ }^{1}$, Manish Soneja², Karan Madan ${ }^{3}$.

Affiliation: ${ }^{2}$ Department of Microbiology, ${ }^{2}$ Department of Medicine, ${ }^{3}$ Department of Pulmonary Medicine, All India Institute of Medical Sciences, New Delhi, India.

\section{Introduction}

- Scedosporium apiospermum is a ubiquitous fungus.

- The clinical importance of this fungus is mostly seen with immunocompromised host and rarely in immunocompetent patients.

- It can present with wide variety of clinical spectrum involving skin \& soft tissue (most common), central nervous system (CNS), lungs, eye, sinuses or other organs of the body including thyroid.

- Many cases are associated with exposure to water bodies like swimming, near drowning or drowning.

\section{Objectives}

- The primary objective of this study was to ascertain the spectrum of infections, predisposing factors, treatment and outcomes of patients suffering from Scedosporium apiospermum infections attending outpatient clinics or admitted to our hospital.

\section{Methods}

- This was a retrospective study of three years from January 2015 to December 2017.

- All cases of infections due to Scedosporium apiospermum were included in the present study.

- Demographic characteristics, clinical and laboratory data, and mycological examinations were analyzed. Identification was based on culture characteristics and microscopic identification (Fig 1a \& b).

- Antifungal susceptibility was performed on four isolates according to CLSI document M38-A3 for moulds.

\section{Results}

- During the study period, a total of eight cases were identified.

- The mean age was 49 years (range of 2-78 years).

- There were five male and three female patients.

- Five of the eight cases presented with pulmonary symptoms.

- Of these two patients were known cases of HIV (2/5), two had COPD being treated with steroids (2/5) and one case had silicosis (1/5) (Table 1).

- There were two cases of invasive sinusitis and both the patients had no underlying condition.
- There was one case was of B cell acute lymphoblastic leukemia (ALL) who presented with acute suppurative otitis media with mastoiditis.

- Four S. apiospermum isolates tested had MICs in the range of $4-8 \mathrm{mg} / \mathrm{L}$ for amphotericin $\mathrm{B}, \geq 64 \mathrm{mg} / \mathrm{L}$ for fluconazole, $\geq 16 \mathrm{mg} / \mathrm{L}$ for itraconazole and $0.25-0.5 \mathrm{mg} / \mathrm{L}$ for voriconazole, $0.5-1 \mathrm{mg} / \mathrm{L}$ for posaconazole, $\geq 64 \mathrm{mg} / \mathrm{L}$ for flucytosine, $\leq 0.015-0.06 \mathrm{mg} / \mathrm{L}$ for caspofungin and $0.015-0.125 \mathrm{mg} / \mathrm{L}$ for micafungin.

- Six of the eight cases were treated with voriconazole and had a successful outcome. One patient of B cell ALL was given AmB for wider coverage, however succumbed to the illness. Another patient was lost to follow up.

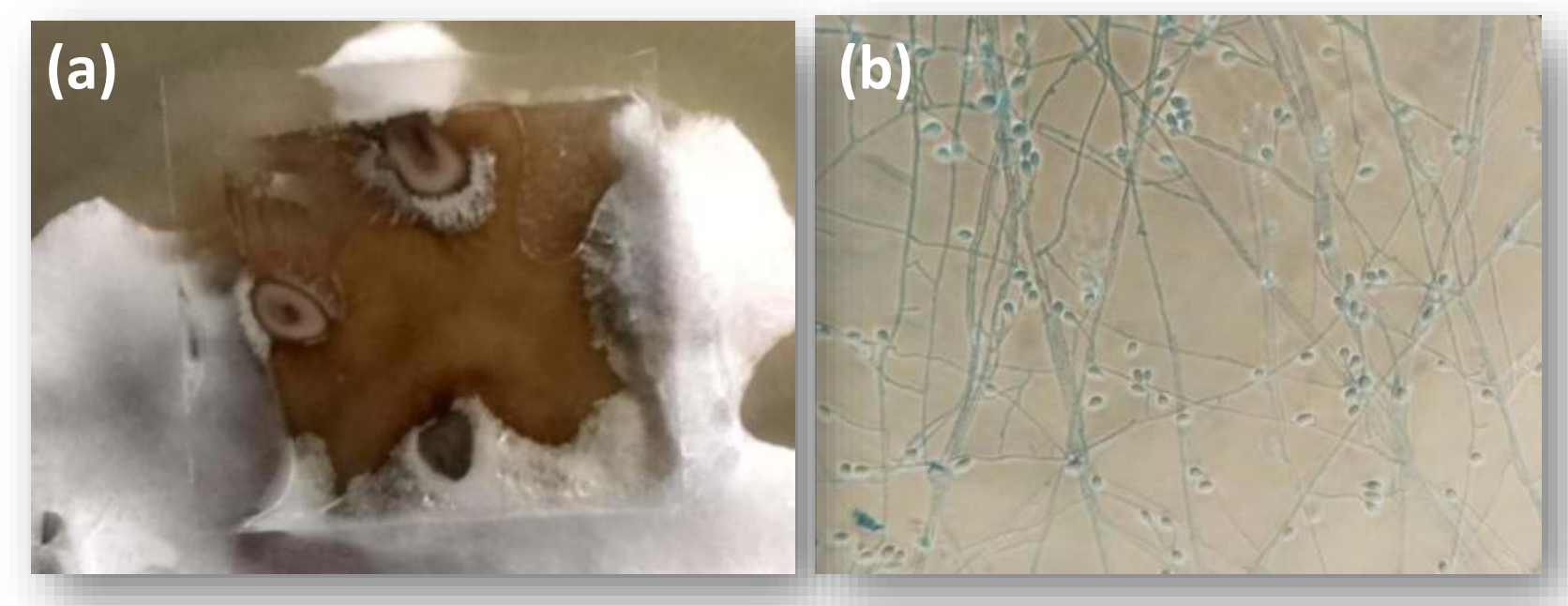

Fig 1 (a). Microculture of an isolate showing greyish-white, suede-like to downy colony morphology. Fig 1 (b) Numerous single-celled, broadly clavate to ovoid conidia, rounded above with truncate bases are observed. Conidia are borne singly or in small groups on elongate, simple or branched conidiophores or laterally on hyphae.

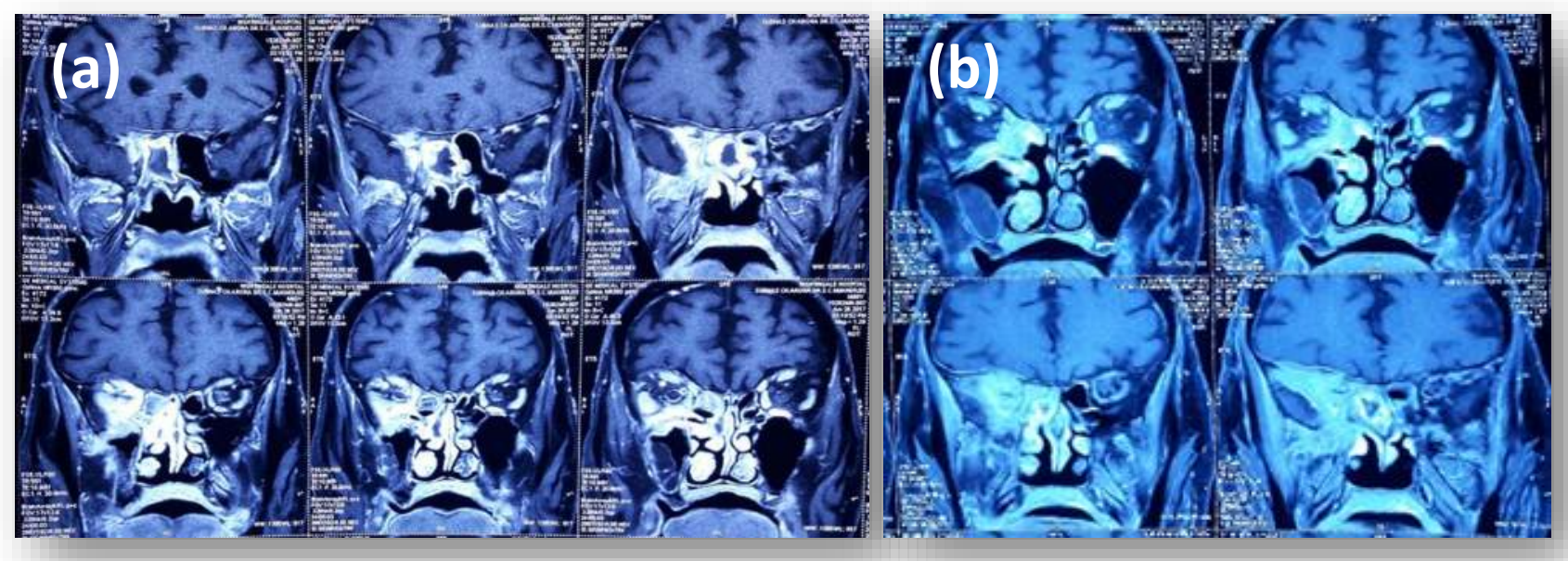

Fig 2. (a)- MRI of patient no. 8 showing enhancement of maxillary sinus, ethmoid sinus, sphenoid sinus and orbit on right side. Fig 2(b)- MRI showing significant resolution after treatment.

\section{Conclusion}

- Infections due to Scedosporium apiospermum are on the rise.

- An early diagnosis helps in initiation of accurate therapy and successful management.

\begin{tabular}{|c|c|c|c|c|c|c|c|c|}
\hline S.No. & Age & Sex & Predisposing Factors & Clinical Presentation & Sample & $\mathrm{KOH}$ & Treatment & Outcome \\
\hline 1 & $70 y$ & M & COPD on long term steroids & Respiratory distress & Sputum & - & - & Unknown \\
\hline 2 & $44 y$ & M & Silicosis & Fever with haemoptysis & BAL & + & Voriconazole & Survived \\
\hline 3 & $63 y$ & $\mathrm{~F}$ & COPD on long term steroids & Respiratory distress & BAL & - & Voriconazole & Survived \\
\hline 4 & $02 y$ & $\mathbf{F}$ & B cell ALL with induction therapy & ASOM with mastoiditis & Nasal debris & + & AmB & Died \\
\hline 5 & $40 y$ & M & HIV positive & Prolonged cough & BAL & - & Voriconazole & Survived \\
\hline 6 & $30 y$ & $\mathbf{F}$ & HIV positive & Prolonged cough & BAL & - & Voriconazole & Survived \\
\hline 7 & $78 y$ & M & Nil & Sinusitis & Sphenoid biopsy & + & Voriconazole & Survived \\
\hline 8 & $65 y$ & $\mathbf{M}$ & Nil & Sinusitis & Maxillary bone & + & Voriconazole & Survived \\
\hline
\end{tabular}

Table 1: Demographic characteristics, clinical and laboratory data of patients with Scedosporium apiospermum infection 